\title{
Thermal Modelling of Quantum Cascade Lasers
}

\author{
V. Spagnolo ${ }^{a, b, *}$, M.S. Vitiello ${ }^{b}$, C. Di Franco ${ }^{a, b}$ And G. Scamarcio ${ }^{a, b}$ \\ ${ }^{a}$ CNR-INFM Regional Laboratory LIT, Bari, Italy \\ ${ }^{b}$ Dipartimento Interateneo di Fisica "M. Merlin", Università degli Studi e Politecnico di Bari, Bari, Italy \\ One of the top priorities in the development of quantum cascade laser sources is the optimization of \\ the heat transport dynamic. We review here our experimental studies on the thermal properties of state of \\ art quantum cascade lasers operating both in the mid-IR and $\mathrm{THz}$ ranges. The experimental approach is \\ based on the investigation of the band-to-band photoluminescence signals, collected during device continu- \\ ous wave operation. We measured the lattice temperature profile on the device front facet and using these \\ data as inputs, we extract the heat dissipation patterns, the in-plane and the cross-plane active region thermal \\ conductivities and the thermal boundary resistance for quantum cascade lasers based on different material systems.
}

PACS numbers: $61.46 .-\mathrm{w}, 61.82 . \mathrm{Fk}, 68.35 . \mathrm{Ct}$

\section{Introduction}

Quantum cascade lasers (QCLs) are unipolar semiconductor lasers based on electron resonant tunnelling and optical transitions between quantized conduction band states. Quantum engineering of the electronic energy levels and tailoring of the wave functions are used to obtain the correct matrix elements and scattering rates which enable population inversion and the optimization of the overall laser performance. The laser wavelength can be selected by tailoring the active layer thickness [1]. Laser emission has been reported in a wide range of mid-infrared wavelengths $(3.0-24 \mu \mathrm{m})$ and terahertz range $(60-441 \mu \mathrm{m})[2,3]$. Excellent performance in the mid-IR range in terms of peak power $(12 \mathrm{~W}$ at room temperature) [4] and maximum operating temperature $(\approx 470 \mathrm{~K})$ have been achieved in pulsed mode. Continuous wave (cw) operation up to $330 \mathrm{~K}$ has been demonstrated. Output power up to $2.8 \mathrm{~W}$ have been measured at room temperature (RT) with wall-plug efficiency of $15 \%$ [5], while at $80 \mathrm{~K} 10 \mathrm{~W}$ of power output and a wall-plug efficiency of $33 \%$ have been demonstrated [6].

Compared to the mid-IR, QCLs at much longer wavelengths face unique challenging issues and because of the narrow separation between subband levels, heating and electron-electron scattering will have a much greater effect. The highest reported operating temperature for GaAs/AlGaAs THz QCLs is $225 \mathrm{~K}$ in pulsed mode [3] and $117 \mathrm{~K}$ in cw, the highest wall-plug efficiency reported is $\approx 5.5 \%[7]$.

Although these improvements, the lifetime for QCLs under cw operation is still much lower than that of quantum well diode lasers. This is mostly due to the high elec-

\footnotetext{
* corresponding author; e-mail: spagnolo@fisica.uniba.it
}

trical power needed to achieve the laser threshold since the cascading scheme inherently requires applied threshold voltages of several volts. Moreover, the QCLs device thermal resistance is strongly increased by the ternary alloy nature of employed materials, the presence of a large number of interfaces and the associated phonon interference effects [8]. These characteristics leads to a temperature of the active material much greater than the heat sink. Thus, the attainment of device operation in a wider range of wavelengths and the enhancement of the maximum operating temperature requires a deeper knowledge on the key physical phenomena controlling thermal dissipation in QCLs.

Due to its superlattice-like nature, the thermal conductivity of the QCL active region is strongly anisotropic and as a result, both the in-plane $\left(k_{\|}\right)$and cross-plane thermal conductivities $\left(k_{\perp}\right)$ are reduced compared to the bulk values of the constituent materials [9]. Since the layer widths are comparable to or less than the phonon mean free path, the phonon scattering rate at the interfaces between layers increases, hindering the phonon transport. The partially diffuse scattering of phonons at the interfaces can explain the reduction in $k_{\|}$, while the stronger reduction in $k_{\perp}$ is caused by the multiple reflections of phonons at the many interfaces. Furthermore, THz QCLs particularly suffer compared to MIR devices since they generally contain more active region periods resulting in a larger number of interfaces and a higher value of thermal resistance $[7,10,11]$.

To address all these issues we have developed a $\mu$-probe photoluminescence technique allowing us to measure the facet lattice temperature profiles in QCLs under cw operation $[8,9]$.

The obtained experimental data have been reproduced using an anisotropic thermal model taking into account the effect of temperature and doping on the thermal con- 
ductivity of the different bulk-like layers composing the QCL and leaving the in-plane $\left(k_{\|}\right)$and the cross-plane components of the active region thermal conductivity tensor as the only fitting parameters. Using this method we validated the heat diffusion model and directly extracted the heat dissipation patterns and used the model as a workbench to compare the thermal properties of QCLs having the same gain medium, but different heat sinking configurations [12].

The paper is organized as follows. The experimental procedure is outlined in Sect. 2. In Sect. 3, the development of steady-state thermal models of heat diffusion in QCLs are described, with an overview of the results obtained and conclusions from our results.

\section{Experimental procedure}

The photoluminescence (PL) signal was obtained by focusing the line of a $\mathrm{Kr}^{+}$laser directly onto the QCL front facet down to a spot of $\approx 1 \mu \mathrm{m}$ diameter by using an $80 \times$ long-working-distance microscope objective lens (see Fig. 1). The incident power density was kept $<10^{4} \mathrm{~W} / \mathrm{cm}^{2}$ to avoid laser-induced heating. The device was mounted into a helium-flow micro-cryostat. The heat sink temperature $\left(T_{\mathrm{H}}\right)$ was measured with a calibrated Si-diode mounted close to the device.

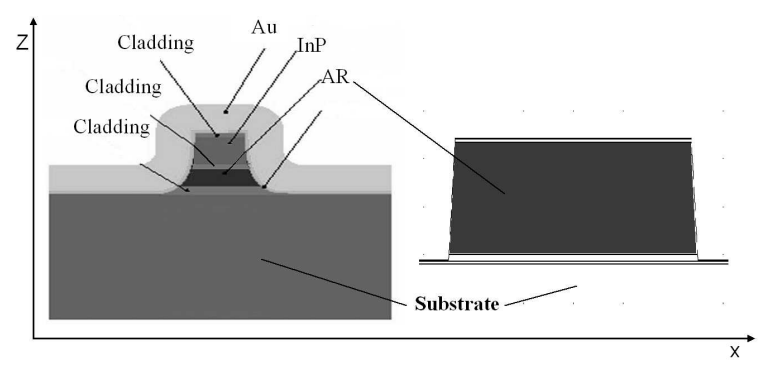

Fig. 1. Schematics of a typical mid-IR and THZ QCL front facet, the black areas represent the related active regions (AR) layer.

The sample position relative to the $\mathrm{Kr}^{+}$laser was varied by a two-dimensional piezoelectric translation stage with $0.1 \mu \mathrm{m}$ spatial resolution. The PL signal was dispersed using a monochromator and detected by a standard $\mathrm{Si}$-CCD or a $\mathrm{N}_{2}$-cooled GaInAs diode array detector. The use of the GaInAs detector is required for detection of PL signal falling in the wavelength range below $1 \mu \mathrm{m}$, due to the long-wavelength cut-off of Si-based detection system.

The shift of the PL spectra was used as a thermometric property. By probing the device at zero current while varying the heat sink temperature $T_{\mathrm{H}}$ we determined the PL peak position as a function of the lattice temperature, thereby obtaining calibration curves for the different regions composing the devices in good agreement with known band-gap temperature dependences. Then, we kept fixed the heat sink temperature and varied the
QCLs electrical power. The laser facet temperature profiles were measured by comparing the shift of the PL peak with the calibration curves. In the case of PL emitted from the quantum well active region, electric field effects were taken into account, following the method outlined in Ref. [13]. We were able to determine the thermal gradient both along the $X$ axis and the growth axis $Z$ (see Fig. 1). The temperature resolution for the different layers composing the devices depends on related energy gap temperature dependence, typically we obtained temperature resolution $\leq 0.7 \mathrm{~K}$.

\section{Steady-state thermal model}

The device lattice temperature distribution and the heat flux pattern have been calculated using a two-dimensional anisotropic steady-state heat dissipation model based on the Fourier law of heat conduction

$$
-\nabla(k \cdot \nabla T)=Q,
$$

where $Q$ is the heat source, $T$ is the temperature and $k$ is the thermal conductivity tensor.

The only fitting parameter in this model is the anisotropic thermal conductivity. We used temperature dependent values for the heat conductivities of the different layers composing the investigated devices. In Fig. 2 there is shown a selection of thermal conductivity values for materials typically employed in the realization of QCLs.

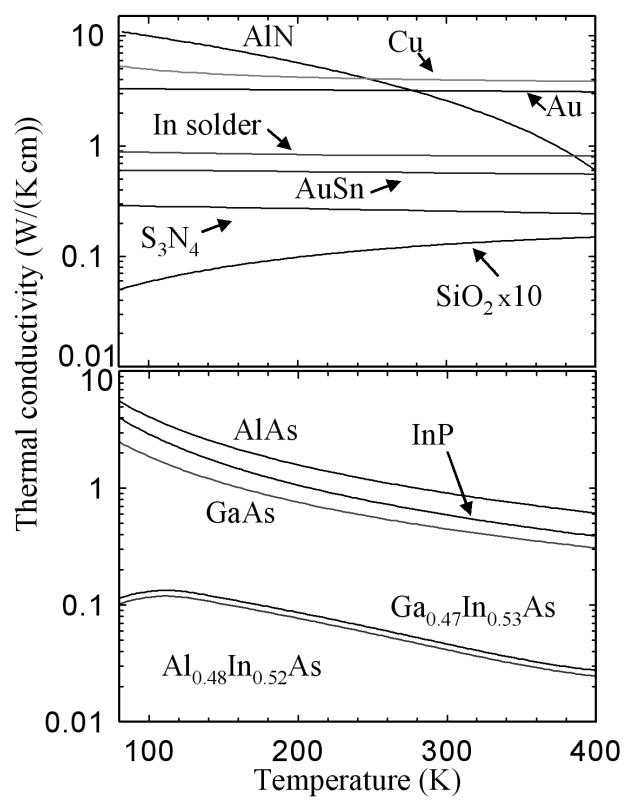

Fig. 2. Temperature dependence of the thermal conductivity of different materials composing the investigated QCLs.

In the calculations we included the effect of doping that reduces the semiconductor thermal conductivity by $8 \%$ for each increasing decade of electrically active impurities concentration [14], starting from $10^{15} \mathrm{~cm}^{-3}$. As 
boundary conditions we used the experimental heat sink temperature, with the assumption that no heat escape through the sides or top of the laser exists.

The comparison of the experimental temperature profiles with the calculations allow us to validate the model. The model can thus be used to determine the 2D-temperature profile.

One of the outcomes of the model is the heat dissipation pattern of the devices, which strongly depends from the device geometry and mounting configurations. In Fig. 3 there are shown two representative heat dissipation patterns calculated for a junction-up mid-IR QCLs with thick gold layer on top and a bound-to-continuum THz QCL.
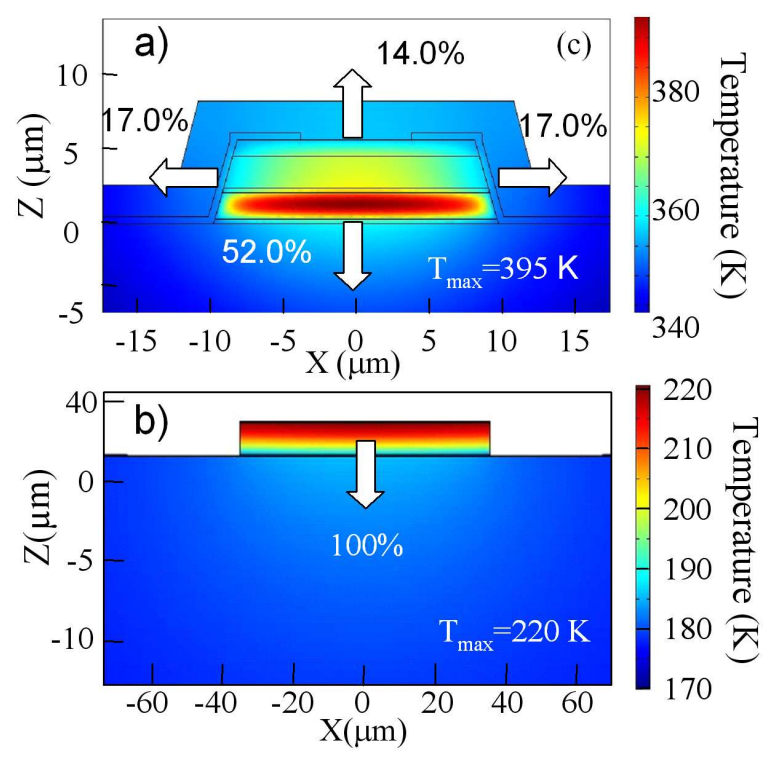

Fig. 3. (a) Heat flux configuration for a junction-up InGaAs/AlInAs/InP mid-IR QCL with $2 \mu \mathrm{m}$ thick gold layer on top; (b) heat flux configuration for a bound-to-continuum GaAs/AlGaAs THz QCL.

The strong differences in the heat flow configuration is manly due to the different device geometry between mid-IR and THZ QCLs. In mid-IR QCLs, the ridges are narrow (typically down to few $\mu \mathrm{m}$ ), surrounded by an insulated layer and by an Au contact layer, the devices may be buried by means of InP re-growth and mounted epilayer-up or -down. The consequences of these characteristics are:

1. even if it is not very efficient, the insulated layer is a lateral channel for the heat flow;

2. employing a thick top contact layer greatly improves the heat extraction through the top cladding and leads also to significant lateral heat fluxes, thus facilitating the heat extraction in all in-plane directions;

3. in buried device there is strongly enhanced the lateral heat flow;
4. the use of an epilayer-down mounting greatly improves heat dissipation since it reduces the thermal path between the heat source (active region) and the sink.

In $\mathrm{THz}$ QCLs, the absence of layers surrounding the ridge prevents heat from flowing from the top towards the substrate and laterally. However, in THz QCLs the absence of the lateral channels has no great consequences with regard to the heat management, when we consider device with large width of the ridges $(>150 \mu \mathrm{m})$. Moreover, in THz QCLs, $k_{\perp}$ can be easily extracted from the experimental data, taking advantage of the absence of lateral heat extraction channels. In fact, under this condition and assuming that in QCLs the heat is uniformly dissipated in the whole active region, the lattice temperature distribution across the active region is given by a polynomial of the second order. Thus, we can extract the cross-plane component of the active region thermal conductivity simply from the quadratic fit of the experimental data.

The cross-plane thermal conductivity parameter depends on the material systems employed and also on the number of layer composing the active region and can be expressed as a function of the bulk resistivities of the materials $\left(R_{i}\right)$ and the average thermal boundary resistance (TBR) that keeps into account the mean contribution of the interface resistivities. The following relation holds [15]:

$$
\left(k_{\perp}\right)^{-1}=\sum_{i} \frac{d_{i}}{d_{\mathrm{tot}}} R_{i}+\frac{N}{d_{\mathrm{tot}}} \mathrm{TBR},
$$

where $d_{i}$ is the thickness of the well, or barrier layers in the active region, $d_{\text {tot }}$ the total active region thickness and $N$ the total number of interfaces [16]. In QCLs the bulk resistivity values have a negligible effect on the thermal conductivity of active regions. In fact, the latter is mostly determined by the high density of interfaces and hence by the second term in the above expression for $\left(k_{\perp}\right)^{-1}$.

TABLE Cross-plane thermal conductivity and thermal boundary resistance in a set of quantum cascade lasers, classified on the basis of the emitted wavelength and material system.

\begin{tabular}{c|c|c|c|c}
\hline \hline Device material system & $\lambda[\mu \mathrm{m}]$ & $\mathrm{T}[\mathrm{K}]$ & $\begin{array}{c}k_{\perp} \\
{[\mathrm{W} /(\mathrm{K} \mathrm{m})]}\end{array}$ & $\begin{array}{c}\mathrm{TBR} \\
{\left[10^{9} \mathrm{~m}^{2} \mathrm{~K} / \mathrm{W}\right]}\end{array}$ \\
\hline InGaAs/AlInAs & 8 & 80 & 1.7 & 4.4 \\
InGaAs/AlGaAsSb & 4.9 & 60 & 2.0 & 0.58 \\
InGaAs/AlInAs & 4.8 & 60 & 2.0 & 0.50 \\
$\mathrm{GaAs} / \mathrm{Al}_{0.33} \mathrm{Ga}_{0.67} \mathrm{As}$ & 9.4 & 90 & 5.5 & 0.51 \\
$\mathrm{GaAs} / \mathrm{Al}_{0.15} \mathrm{Ga}_{0.85} \mathrm{As}$ & 105 & 45 & 5.4 & 1.28 \\
$\mathrm{GaAs} / \mathrm{Al}_{0.15} \mathrm{Ga}_{0.85} \mathrm{As}$ & 106 & 45 & 8.1 & 1.35
\end{tabular}

In Table there are reported the TBR values and the relative cross plane thermal conductivities, measured in a set of mid-IR and $\mathrm{THz}$ QCLs based on 
GaAs/AlGaAs, GaInAs/InAlAs and GaInAs/AlGaAsSb material systems.

Our results show that the relative weight of the interface resistivity is dominant with respect to the bulk-like ones, thus demonstrating that the thermal properties of QCLs are primarily controlled by nanoscale heat transport at the interfaces.

Future developments include the study of the influence of interface structure on the thermal boundary resistance and the exploitation of the thermal model for the development of new strategies for the realization of QCL having lower thermal conductivities.

\section{References}

[1] C. Gmachl, F. Capasso, D.L. Sivco, A.Y. Cho, Rep. Prog. Phys. 64, 1533 (2001).

[2] R. Kohler, A. Tredicucci, F. Beltram, H.E. Beere, E.H. Linfield, A.G. Davies, D.A. Ritche, R.C. Iotti, F. Rossi, Nature 417, 156 (2002).

[3] A. Wadel, G. Fedorov, D. Smirnov, S. Kumar, B.S. Williams, Q. Hu, J.L. Reno, Nature Photon. 3 41 (2008).

[4] Y. Bai, B. Gokden, S.R. Darvish, S. Slivken, M. Razeghi Appl. Phys. Lett. 95, 031105 (2009).

[5] M. Razeghi, IEEE J. Select. Top. Quant. Electr. 15, 941 (2009).

[6] Y. Bai, B. Gokden, S. Slivken, S.R. Darvish, S.A. Pour, M. Razeghi, Proc. SPIE 7222, $72220 \mathrm{O}$ (2009).
[7] M.S. Vitiello, G. Scamarcio, V. Spagnolo, S.S. Dhillon, C. Sirtori, Appl. Phys. Lett. 90 191115 (2007).

[8] M.S. Vitiello, G. Scamarcio, V. Spagnolo, IEEE J. Select. Top. Quant. Electr. 14, 431 (2008).

[9] A. Lops, V. Spagnolo, G. Scamarcio, J. Appl. Phys. 100, 043109 (2006).

[10] M.S. Vitiello, G. Scamarcio, V. Spagnolo, B.S. Williams, S. Kumar, Q. Hu, J.L. Reno, Appl. Phys. Lett. 86, 111115 (2005).

[11] M.S. Vitiello, G. Scamarcio, V. Spagnolo, J. Alton, S. Barbieri, C. Worral, H.E. Beere, D.A. Ritchie, C. Sirtori, Appl. Phys. Lett. 89, 021111 (2006).

[12] V. Spagnolo, A. Lops, G. Scamarcio, M.S. Vitiello, C. Di Franco, J. Appl. Phys. 103, 043103 (2008).

[13] V. Spagnolo, G. Scamarcio, D. Marano, M. Troccoli, F. Capasso, C. Gmachl, A.M. Sergent, A.L. Hutchinson, D.L. Sivco, A.Y. Cho, H. Page, C. Becker, C. Sirtori, IEEE Proc. Optoelectron. 150, 298 (2003).

[14] J.C. Brice, EMIS Datareviews Series No. 6, INSPEC, London 1991.

[15] M.S. Vitiello, G. Scamarcio, V. Spagnolo, A. Lops, Q. Yang, C. Manz, J. Wagner, Appl. Phys. Lett. 90, 121109 (2007).

[16] G. Scamarcio, M.S. Vitiello, V. Spagnolo, S. Kumar, B. Williams, Q. Hu, Physica E 40, 1780 (2008). 